\title{
Determinación de la difusividad térmica en sólidos mediante el análisis de la amplitud de la señal fotoacústica en configuración de transmisión de calor.
}

\begin{abstract}
G. Peña-Rodríguez ${ }^{1, *}$, A. Calderón ${ }^{2}$ y R. A. Muñoz-Hernández ${ }^{2}$
${ }^{1}$ Departamento de Física, Universidad Francisco de Paula Santander. A.A. 1055, Cúcuta, Norte de Santander, Colombia.

${ }^{2}$ Centro de Investigación en Ciencia Aplicada y Tecnología Avanzada del Instituto Politécnico Nacional, Legaria 694 Colonia Irrigación, 11500 México D. F.
\end{abstract}

\section{Resumen}

En este trabajo, presentamos el desarrollo teórico en configuración de transmisión de calor en la celda fotoacústica, el cual es usado para la determinación experimental de la difusividad térmica de sólidos, dicho estudio se presenta tomando como base el del modelo de Rosencwaig y Gersho (RG), el cual utiliza el mecanismo de difusión térmico como el principal generador de la señal fotoacústica (FA). El aporte de este trabajo es la presentación de la expresión para la amplitud de la señal FA en función de la frecuencia de modulación de la radiación incidente, en la configuración de transmisión de calor, la cual es utilizada para ajustar los datos experimentales y así obtener la difusividad térmica de los materiales a estudiar. También se presenta la aproximación para el régimen térmicamente grueso y se reportan los rangos de validez de esta aproximación en función de la frecuencia de corte o característica de cada material.

Palabras claves: difusividad térmica, transferencia de calor, técnicas fototérmicas.

\section{Introducción}

Dentro de los fenómenos físicos observados en la interacción radiación materia, encontramos aquellos en los cuales la energía absorbida es transformada vía procesos de desexcitación no radiativos en calor. Sí colocamos un material dentro de una celda herméticamente cerrada y hacemos incidir luz modulada sobre éste, el material absorbe la radiación incidente y genera en su interior una respuesta térmica, la cual perturba el gas de sus alrededores produciendo una variación de la presión en la celda y generando una señal acústica, este fenómeno es llamado efecto fotoacústico y fue descubierto en 1880 por A. G. Bell.

Noventa y seis años después Rosencwaig y Gersho (1976) [1], explicaron por primera vez, teórica- mente el efecto fotoacústico, basados en el tratamiento de ondas térmicas. Su hipótesis supone que una delgada capa de aire se expande y contrae en la interface airesólido dentro de la celda, la cual actúa sobre el resto del volumen, produciendo cambios en la presión de éste y generando la señal acústica. El estudio de esta señal fue usado por Rosencwaig para establecer la espectroscopía fotoacústica (EFA), con el objetivo de estudiar propiedades ópticas en sólidos [2].

Desde su presentación hecha en 1977 por Adams y Kirkbright [3], hasta nuestros días, la técnica fotoacústica en configuración de transmisión de calor se ha utilizado cada vez con mayor frecuencia en la medición de una amplia variedad de materiales [4-7]. Esta técnica ha probado ser atractiva debido a que combina la aplicación "sin contactos" de bien definidas cantidades de energía térmica con métodos sensibles para la determinación de los campos de temperatura resultantes. Además, como requiere pequeñas cantidades de muestra a analizar, permite la determinación de la difusividad térmica en regiones localizadas del material.

La difusividad térmica es la propiedad física que mide la razón de flujo de calor a través de un medio, la importancia de esta cantidad física se debe a que al igual que el coeficiente de absorción óptico es única para cada material [8]. La difusividad térmica es una cantidad extremadamente sensible a la composición y estructura de los materiales [6]. Este es particularmente el caso de las propiedades termofísicas en los materiales porosos, las cuales dependen fuertemente además de la clase de componentes constituyentes, del tipo de estructura que presentan y de su grado de porosidad [9].

Por lo anterior, en este trabajo presentamos una descripción del diseño experimental de la técnica FA y de la configuración de transmisión de calor de la celda FA, utilizadas para hallar experimentalmente la difusividad térmica de materiales sólidos. Luego se presenta un desarrollo teórico del modelo RG usado por la técnica FA. Tomando como base el modelo RG, se realiza un desarrollo teórico teniendo en cuenta la configuración de transmisión de calor de la celda FA, para reporta la expresión de la amplitud de la señal FA en función de la frecuencia de modulación de la radiación incidente en ésta configuración, expresión que permitirá del ajuste de los datos experimentales hallar la difusividad térmica de la muestra a estudiar, también, se presenta un análisis de ésta expresión en los regímenes térmicamente delgado y térmicamente grueso, así como los rangos de validez de 
dichas aproximaciones en función de la frecuencia de corte del material.

\section{Diseño experimental de técnica FA y configu- ración de transmisión de calor de la celda FA.}

En la figura 1, se muestra el montaje experimental utilizado en la detección de las ondas térmicas por la técnica FA. El haz del láser se interrumpe periódicamente mediante un modulador mecánico de rapidez variable (chopper), el haz así modulado se desvía por un espejo y se hace incidir en dirección normal sobre la superficie de la muestra, la cual previamente se ha colocado en la entrada de la celda fotoacústica (CFA) adherida con pasta térmica o grasa de vacío. La señal acústica genera-da en el interior de la celda es detectada por un micrófono de alta sensibilidad. Luego, la señal eléctrica resultante es capturada y filtrada con la frecuencia de referencia del chopper mediante un amplificador lock-in. Finalmente, se registra en la computadora un archivo de la amplitud y la fase de la señal FA en función de la frecuencia de modulación.

La CFA en configuración de transmisión de calor se presenta en la figura 2, la cual es utilizada para la medida de la difusividad térmica en sólidos. La muestra se coloca adherida con grasa de vacío o pasta térmica en la parte superior de la celda, de manera que una de las caras de la muestra (cara delantera) esta en contacto con el aire en el interior de la celda y la otra cara (cara trasera) da al exterior de la misma. Si se hace incidir radiación modula-da en la cara trasera de la muestra, la energía absorbida por ésta genera una respuesta térmica que se transmite a través de la muestra y se difunde al interior de la cámara FA dando lugar a fluctuaciones de presión que son detectadas por un micrófono de alta sensibilidad.

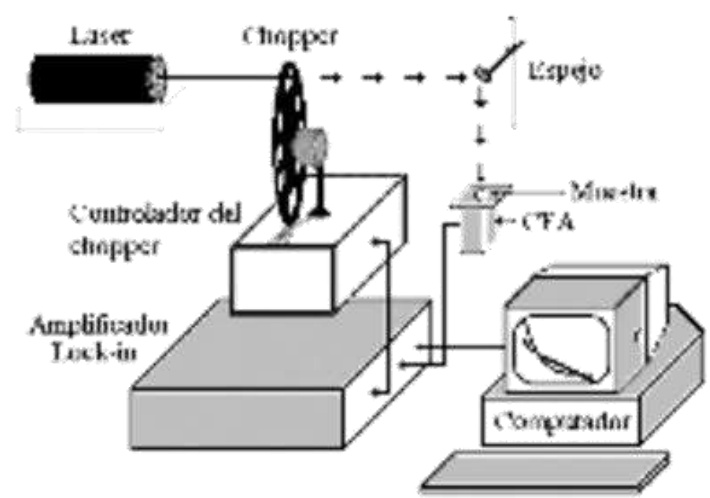

Figura 1. Diseño experimental de la técnica fotoacústica.

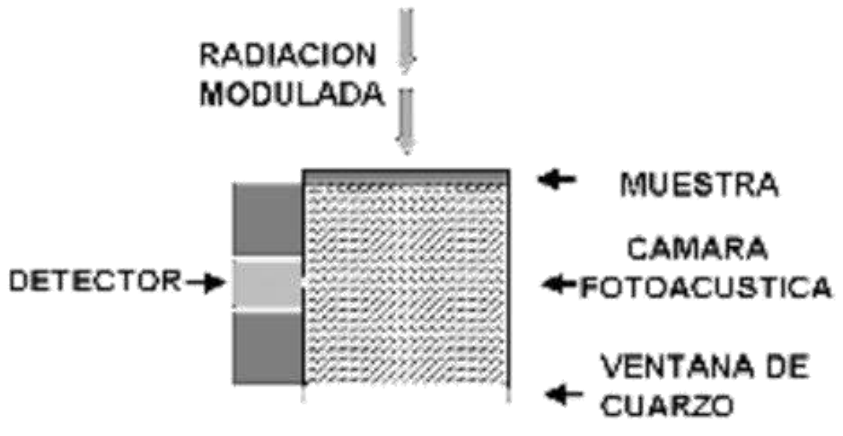

Figura 2. Corte transversal de la CFA en configuración de transmisión de calor.

\section{El modelo RG.}

En este modelo teórico se considera al mecanismo de difusión térmico como el principal responsable de la producción de la señal fotoacústica (FA) [1]. A través del estudio unidimensional del flujo de calor en la celda(ver fig. 3), Rosencwaig y Gersho demostraron que solamente una capa delgada de aire de unas décimas de centímetro de espesor adyacente a la superficie del sólido, responde térmicamente al flujo de calor periódico proveniente del sólido. Esta capa de aire sufre un calentamiento y un enfriamiento alternado y se comporta como un pistón vibratorio, el cual genera la señal fotoacústica.

Consideremos una celda cilíndrica de diámetro $D$ y longitud $L$, como se muestra en la Figura 3 . En ésta figura $l$ es el espesor de la muestra, la cual está en su parte frontal expuesta al gas de la celda y la superficie trasera se encuentra en contacto con la base de espesor $l_{b}$. En esta misma figura se observa que la longitud de la

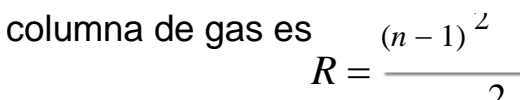

$$
(n+1)^{2}
$$

Suponemos también que la celda y el gas no absorben radiación.

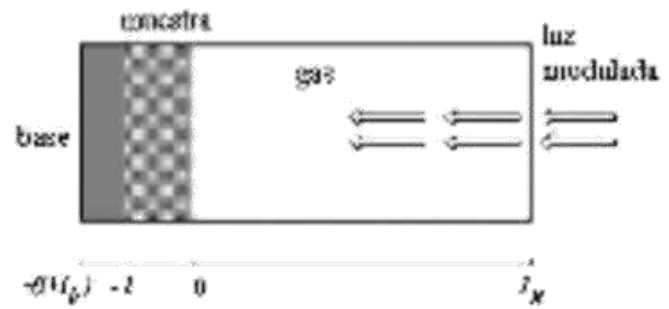


Figura 3. Sección transversal de la CFA convencional usada en el modelo RG.

Tabla 1. Algunos parámetros termofísicos que serán uti-lizados en este trabajo.

\begin{tabular}{lll}
\hline$k i$ & $\begin{array}{l}\text { Conductividad térmica material } \\
i .\end{array}$ & $(\mathrm{W} / \mathrm{m} \mathbf{K})$ \\
\hline$\rho i$ & Densidad de masa material $i$. & $\left(\mathbf{K g} / \mathrm{m}^{3}\right)$ \\
\hline$c_{i}$ & Calor especifico material $i$. & $(\mathrm{J} / \mathrm{g} \mathbf{K})$ \\
\hline$\alpha i=k i / \rho i c i$ & Difusividad térmica material $i$. & $\left(\boldsymbol{m}^{2} / \mathbf{s e g}\right)$ \\
\hline$a i=\omega \sqrt{2 \alpha} i$ & $\begin{array}{l}\text { Coeficiente de difusión térmico } \\
\text { material } i .\end{array}$ & $\left(\boldsymbol{m}^{-1}\right)$ \\
\hline$\mu_{i}=1 / a i$ & $\begin{array}{l}\text { Longitud de difusión térmica } \\
\text { material } i .\end{array}$ \\
\hline$\sigma i=(1+j) a_{i}$ & $\begin{array}{l}\text { Coeficiente de difusión térmico } \\
\text { complejo material } i .\end{array}$ & $\left(\boldsymbol{m}^{-1}\right)$ \\
\hline
\end{tabular}

En la tabla 1, se definen algunos parámetros que serán usados durante el desarrollo de este trabajo. El subíndice $i$ denota la muestra $(s)$, el gas $(g)$ o la base $(b), \omega=2 \pi /$ es la frecuencia de modulación en rad/seg y $j=/ \neg 1$.

Analicemos la ecuación de difusión de calor para cada región de la Figura 3, donde la luz que proviene de la derecha atraviesa el gas no absorbente $g$ de espesor $l_{g}$, e incide en $x=0$ sobre la superficie de la muestra $s$ de espesor $l$, creando en su interior una fuente de calor $g(x, t)$, luego:

$$
\begin{aligned}
& \frac{\partial^{2}}{\partial x^{2}} \Theta_{g}(x, t)-\frac{1}{\alpha_{g}} \frac{\partial}{\partial t} \Theta_{g}(x, t)=0 \\
& \frac{\partial^{2}}{\partial x^{2}} \Theta_{S}(x, t)-\frac{1}{\alpha_{s}} \frac{\partial}{\partial t} \Theta_{s}(x, t)=-g(x, t) \quad ; 0 \leq x \leq l_{g} \\
& \frac{\partial^{2}}{\partial x^{2}} \Theta_{b}(x, t)-\frac{1}{\alpha_{b}} \frac{\partial}{\partial t} \Theta_{b}(x, t)=0 \quad ;-l \leq x \leq 0
\end{aligned}
$$

En las expresiones (1)-(3), $\Theta_{i}$ denota la diferencia de temperaturas entre el medio $i(i=g, s, b)$ y el medio ambiente, por lo tanto $T_{i}(x, t)=\Theta_{i}+T_{0}$ ??representa la temperatura del medio $i$, con $T_{0}$ ?la temperatura ambiente.

La continuidad de la temperatura y el flujo de calor en las interfaces de los medios $g, s$ y $b$, permiten escribir las condiciones de frontera, las cuales están dadas por:

$$
\begin{aligned}
& \Theta_{g}(0, t)=\Theta_{S}(0, t) \\
& \Theta_{b}(-l, t)=\Theta_{s}(-l, t) \\
& k_{g} \frac{\partial}{\partial x_{s}} \Theta(0, t)=k_{s} \frac{\partial}{\partial x} \Theta(0, t) \\
& { }_{b}^{k}{\frac{\partial}{\partial x_{b}} \Theta}_{b}(-l, t)=k{\frac{\partial}{s} \partial x_{s}}_{s}(-l, t)
\end{aligned}
$$

Si se considera que la intensidad del haz de luz monocromático incidente está modulado en el tiempo en la forma:

$$
I(t)=\frac{I_{0}}{2}\left[1+e^{j \omega t}\right]
$$

Donde $I_{0}$ denota la intensidad de la luz monocromática incidente $\left(W / \mathrm{cm}^{2}\right)$, entonces, la fuente de calor que aparece en la ecuación (2), puede escribirse como:

$$
g(x, t)=G\left(x \left[1+e^{j \bar{\phi} t}\right.\right.
$$

Donde $G(x)$ representa la forma en que la radiación es absorbida, y que depende de las propiedades ópticas y térmicas de la muestra, lo cual se tendrá en cuenta más adelante.

La solución de las ecuaciones (1)-(3), con las condiciones de frontera (4)-(7), se componen de una parte estacionaria, que depende solo de la posición y una parte que oscila en el tiempo, luego considerando solo la parte que oscila en el tiempo, que es la encargada de originar las ondas térmicas, las cuales producen el efecto fotoacústico, analizando el problema en forma unidimensional y teniendo en cuenta la ecuación (9), tenemos que la solución de este sistema de ecuaciones con estas condiciones de frontera para la parte que oscila en el tiempo $t_{i}$ están dadas por:

$$
\begin{array}{ll}
\tau_{g(x)=\tau_{s}(0) e^{-\sigma g x}} & ; 0 \leq x \leq l_{g} \\
\tau_{b}(x)=\tau_{s}(-l) e^{\sigma_{g}(x+l)} & ;-(l+l b) \leq x \leq-l
\end{array}
$$




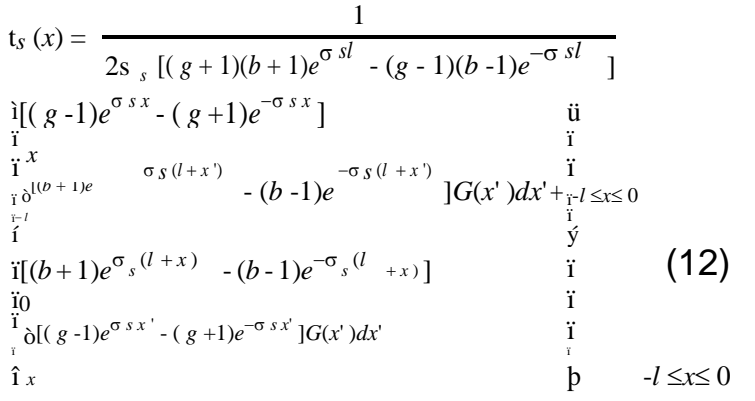

donde $\quad g=\frac{k_{g} \sigma_{g}}{k_{s} \sigma_{s}}=\frac{e_{g}}{e_{s}} \quad \mathrm{y} \quad b=\frac{k_{b} \sigma_{b}}{k_{s} \sigma_{s}}=\frac{e_{b}}{e_{s}}$

Como puede verse en (12), para determinar completamente la solución, es necesario especificar el parámetro $G(x)$.

Considerando que la absorción de la radiación puede darse de dos formas distintas dependiendo de las propiedades ópticas de la muestra, lo que da lugar a dos modelos para la fuente de calor $g(x, t)$ : el modelo de absorción volumétrico (Ley de Beer) y el modelo de fuente superficial. El modelo de absorción de Beer, considera que la radiación incidente se absorbe de tal forma que la intensidad de la fuente de calor generada en el interior del material disminuye exponencialmente con la profundidad. La atenuación de la intensidad de la fuente de calor esta caracterizada por coeficiente de absorción óptico ( $\mathrm{b}$ ) el cual es único para cada material y que depende de la longitud de onda de la radiación incidente. Las expresiones que toma $G(x)$ para cada uno de estos modelos son:

$$
\begin{gathered}
G(x)=\frac{\beta I \eta_{0}}{2 k_{s}} e_{\beta x} \\
G(x)=\frac{\beta I 0 \eta}{2 k_{s}} \delta(x) \quad \text { Absorción volumétrica (14) } \\
\text { Absorción superficial (15) }
\end{gathered}
$$

Donde h representa la razón de la radiación incidente a la radiación absorbida por la muestra.
Sí en (12) hacemos $x=0$ y $x=-l$, con $G(x)$ dado por (14) o (15) según el caso, se podrá cuantificar la magnitud de la señal FA a través de la temperatura de la muestra en sus dos caras, obteniéndose los siguientes resultados:

\section{Absorción Volumétrica (Absorción de Beer)}

$$
\begin{aligned}
& \mathrm{t}_{s}(0)=\frac{\mathrm{b} I_{0} \mathrm{~h}}{2 k_{s}\left(\mathrm{~b}^{2}-\mathrm{s}_{s}^{2}\right)}
\end{aligned}
$$

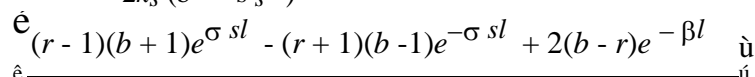

$$
\begin{aligned}
& \hat{\mathrm{e}} \quad(g+1)(b+1) e^{\sigma s l}-(g-1)(b-1) e^{-\sigma s l} \text { ú } \\
& \ddot{\mathrm{e}} \\
& \mathrm{t}_{s}(-l)=\frac{\mathrm{b} I 0 \mathrm{~h}}{2 k_{s}\left(\mathrm{~b}^{2}-\mathrm{s}_{s}^{2}\right)} \\
& \hat{\mathrm{e}} \frac{\mathrm{e}}{2(r+g)-(r+1)(g+1) e^{\sigma s l} e^{-\beta l}+(r-1)(g-1) e^{-\sigma s l} e^{-\beta l}} \mathrm{ù}^{-\sigma} \text { ú }
\end{aligned}
$$

$$
\begin{aligned}
& \text { Absorción Superficial } \\
& \mathrm{t}_{s}(0)=\frac{\mathrm{b} I_{0} \mathrm{~h}}{2 k_{s} \mathrm{~s}_{s}} \\
& { }_{\hat{\mathrm{e}}}^{\mathrm{e}} \frac{(b+1) e^{\sigma s l}-(b-1) e^{-\sigma s l}}{(g+1)(b+1) e^{\sigma s l}-(g-1)(b-1) e^{-\sigma s l}} \text { ú } \\
& \mathrm{t}_{s(-l)}=\frac{\mathrm{b} I_{0} \mathrm{~h}}{2 k_{s} s_{s}} \\
& \text { é } \frac{2}{(g+1)(b+1) e^{\sigma s l}-(g-1)(b-1) e^{-\sigma s l}} \text { ú } \\
& \text { ë }
\end{aligned}
$$

Las expresiones (16) y (17) se reducen a las ecuaciones (18) y (19), sí se consideran materiales ópticamente opacos $\left(\mathrm{b} l_{s} » 1\right)$ y frecuencias de modulación para las que la longitud de difusión térmica es mucho mayor que la longitud de penetración óptica ( $r=\mathrm{b} / \mathrm{s} s \gg 1)$.

La producción de la señal acústica, se debe al mecanismo de difusión térmico que origina una variación periódica en la temperatura del gas, la cual está dada por la componente sinusoidal $(a c)$ de la solución (ver Ecs. (10), y (16)): 


$$
\mathrm{f}_{a c}(x, t)=\mathrm{t}_{s}(0) e^{-\sigma g x+j \omega t}
$$

En (20) notamos que a una distancia de $2 \mathrm{pm}_{\mathrm{g}}$, en la que $\mathrm{m}_{g}$ es la longitud de difusión térmica, la variación periódica de la temperatura en el gas decrece casi totalmente (a un $0.19 \%$ de su valor inicial). Por lo tanto, se puede considerar que una capa de gas de espesor

$2 \mathrm{pm}_{\mathrm{g}}$ adyacente a la superficie de la muestra, responde térmicamente a las variaciones periódicas de la temperatura en la superficie de la muestra. Para frecuencias típicas, de $20 \mathrm{~Hz}$ en adelante, el espesor del pistón térmico toma los valores

$$
2 \mathrm{pm}_{g}=2 \mathrm{p} \quad \sqrt{\frac{\mathrm{a} g}{\mathrm{p} f}} £ 0.35 \mathrm{~cm}
$$

En esta situación, la longitud $l_{g}$ de la capa de aire en la celda debe ser algunas veces mayor que el espesor de este pistón térmico. El dispositivo experimental desarrollado con base a este modelo se denomina CFA convencional o celda cerrada [10]. En esta capa de gas el promedio espacial de la temperatura se puede hallar al calcular la siguiente integral:

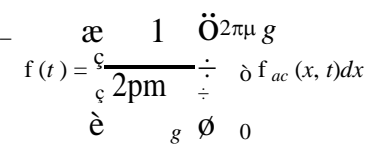

Sustituyendo (20) en (21) y usando la aproximación de que $\mathrm{e}^{-2 \pi}<<1$, tenemos que el promedio espacial de la temperatura esta dado por:

$$
\overline{\mathrm{f}}(t) » \frac{1}{2 \sqrt{2} \mathrm{p}} \mathrm{t} s(0) e^{j(\omega t-\pi / 4)}
$$

Debido al calentamiento periódico ésta capa de gas se expande y contrae periódicamente, lo cual simula un pistón acústico que genera una señal la cual se propaga a través del resto de la columna de gas.

Un a expresión a p roximad a par a e I desplazamiento de este pistón se puede hallar utilizando la ley de los gases ideales:

$$
\mathrm{d} x(t)=2 \mathrm{pm}_{g} \quad \frac{\overrightarrow{\mathrm{f}(t)}}{T_{0}}=\frac{\mathrm{t}_{s}(0) \mathrm{m} g}{\sqrt{2} T_{0}} e^{j(\omega t-\pi / 4)}
$$

$$
\text { Donde } T_{0}=\mathrm{F}_{0}+\mathrm{F}_{E \text {, siendo }} \mathrm{F}_{0 \text { la temperatura }}
$$
ambiente en las paredes de la celda y ${ }_{E}$ el incremento en la temperatura debido a la componente estacionaria del calor absorbido. Además, se ha considerado la temperatura $d c$ (la componente no sinusoidal) promedio de esta capa superficial de gas igual a la temperatura $d c$ en la superficie del sólido.

Ahora bajo la hipótesis de que el resto del gas de la celda responde adiabáticamente a la acción del pistón, podemos hallar el incremento de la presión ya que $P V^{\mathrm{g}}$ $=$ Cte., por lo tanto:

$$
\delta P(t)=\frac{\gamma P_{0}}{V_{0}} \delta V=\frac{\gamma P_{0}}{l_{g}} \delta x(t)
$$

Donde $P_{0}$ es la presión en el medio ambiente, $V_{0}$ el volumen de la celda, $g$ es la razón de calores específicos y - $\delta V$ es el incremento en el volumen. Sustituyendo (23) en (24), se obtiene para el cambio en la presión de la celda acústica,

$$
\begin{aligned}
& \mathrm{d} P(t)=Q e^{j(\omega t-\pi / 4)} \\
& Q=\frac{\mathrm{t}_{s}(0) \mathrm{g} P_{0}}{\sqrt{2} T_{0} l_{g} a_{g}}
\end{aligned}
$$

representa la envolvente compleja de la variación sinusoidal de la presión. La forma explícita de $Q$ se puede obtener reemplazando (16) en (26).

En la ecuación (25), que representa la variación en la presión del gas, se hace difícil su interpretación debido a la complicada expresión que tendrá $Q$. Ahora si hacemos $T_{0} \approx \Phi_{0}$, se puede apreciar que la componente (dc) de la distribución de temperaturas no necesita ser evaluada, por lo tanto la expresión (26) puede ser evaluada por la magnitud y la fase de la onda de presión generada dentro de la celda por el efecto fotoacústico. 


\section{Análisis de la amplitud de la señal FA para medición de la difusividad térmica de sólidos usando configuración de transmisión de calor.}

Comparando la figura 3, la cual se utilizo en el modelo RG, con la figura 2, observamos que podemos utilizar el mismo análisis para la configuración de transmisión de calor que el desarrollado en el modelo RG si consideramos en la figura 2 que la base esta compuesta de aire. Ahora ya no estamos interesados en la solución a la ecuación de difusión en $x=0$, sino en $x=-l$, refiriéndonos a la figura 3 , pues de esta manera determinaremos la distribución de temperatura en la interfase muestra-aire en el interior de la cámara $F A$, en la configuración de transmisión de calor, figura 2. Considerando que la muestra es ópticamente opaca y que la efusividad térmica del aire es mucho menor que la efusividad térmica de la muestra, luego de (13) tenemos que $g \approx 0$ y que $b \approx 0$. Por lo tanto reemplazando en (26) a

t $s(0)$ por t $s(-l)$ el cual para el caso de absorción superficial esta dado por (19), tenemos que $Q$ estará dada por:

$$
\begin{array}{r}
Q=\frac{C}{\mathrm{~s} s} \frac{1}{\mathrm{e}^{\mathrm{e}} e^{\sigma_{s}^{l}}-e^{-\sigma_{s}^{l} \hat{\mathrm{u}}}} \\
\text { con } \quad C=\frac{\mathrm{g} P_{0} \mathrm{~b} I 0 \mathrm{~h}}{k_{s} \sqrt{2} T_{0} l_{g} a_{g}}
\end{array}
$$

Sustituyendo en (28) $\mathrm{a}_{s}^{\mathrm{s}}=(1+j) a_{s \mathrm{y}}$ realizando el álgebra correspondiente se llega a:

$$
Q=\frac{C(m-n)}{2 a_{s}\left(m^{2}+n^{2}\right)}-j \frac{C(m+n)}{2 a_{S}\left(m^{2}+n^{2}\right)}
$$

Con $m$ y $n$ dados por:

$$
\begin{aligned}
& m=\cos \left(a_{s} l\right)\left[e^{a l}-e^{-a l}\right] \\
& n=\operatorname{sen}\left(a_{s} l\right)\left[e^{a_{s} l} \quad+e^{-a_{s} l}\right]
\end{aligned}
$$

De (29) se puede ver tanto la parte real(Re) como la imaginaria( $(\mathrm{Im})$, luego podemos hallar la amplitud de la señal FA usando:

$$
A=\sqrt{(\mathrm{Re})^{2}+(\operatorname{Im})^{2}}
$$

Reemplazando en (32) la parte real $(\mathrm{Re})$ y la parte Imaginaria (Im) de (29), junto con las expresiones dadas para $m$ y $n$, realizando el álgebra respectiva se llega a que la amplitud de la señal FA esta dada por:

$$
\begin{gathered}
A=\frac{\mathrm{g} P_{0} \mathrm{~b} I_{0} \mathrm{~h}}{4 k_{s} T_{0} l_{g}} \frac{1}{a_{g} a_{s}} \frac{1}{\sqrt{\cosh 2\left(a_{s} l\right)-\cos 2\left(a_{s} l\right)}} \\
\quad \text { Ahora como } a_{i}=\sqrt{\frac{\mathrm{p} f}{\mathrm{a}_{i}}} \text { ver tabla 1, entonces la }
\end{gathered}
$$
expresión anterior la podemos escribir como:

$$
A=\frac{C_{0}}{f} \frac{\sqrt{f_{c}}}{\sqrt{\cosh ^{2}\left(\sqrt{f / f_{c}}\right)-\cos ^{2}\left(\sqrt{f f_{c}}\right)}}
$$

Donde $f$ es la frecuencia de modulación de la radiación incidente y $f_{c}$ es la frecuencia de corte o característica de la muestra, la cual es igual a la frecuencia de modulación para la cual la longitud de difusión térmica y el espesor de la muestra son iguales, y $C_{0}$ es una constante dada por:

$$
C_{0}=\frac{\mathrm{g} P O \mathrm{~b} I O \mathrm{~h} l \sqrt{\mathrm{a} g}}{4 k_{S} T O l g}
$$

Luego la expresión (34) es la ecuación para la amplitud de la señal FA en función de la frecuencia $f$ de modulación de la radiación incidente, la cual puede ser utilizada para ajustar los datos experimentales y determinar la frecuencia de corte de la muestra a estudio, y por ende obtener la difusividad térmica(a) del material, ya que la frecuencia de corte $f_{c}$, a y $l$ están relacionadas por la siguiente expresión:

$$
\mathrm{a}=\mathrm{p} f l_{c}
$$

\subsection{Régimen térmicamente fino(RTF).}

Este régimen ocurre para aquellos valores de la 
frecuencia de modulación $f$ en los que el espesor de la muestra es mucho menor que la longitud de difusión térmica $\left(l_{s} \ll \mu_{s}\right)$, es decir, cuando $f$ es mucho menor que la frecuencia de corte $(f \ll f c)$ [1]. Luego utilizando a segundo término la expansión en series para el cosh $(! f$ $\left(f_{c}\right){ }^{\mathrm{y}} \cos \left(l_{\left.f / f_{c}\right)}\right.$ de (34) tenemos:

$$
A \approx \frac{C_{0}}{\sqrt{2}} f_{c} f^{-32}
$$

De (37) se puede observar que la amplitud de la señal FA decrece como $f^{-3 / 2}$, lo cual no permite hallar la difusividad térmica de la muestra al no poderse determinar $f_{c}$ del ajuste de los datos experimentales.

\subsection{Régimen térmicamente grueso(RTG).}

Se le conoce como el régimen térmicamente grueso, a aquellos valores de la frecuencia de modulación $f$ en los que el espesor de la muestra es mucho mayor que la longitud de difusión térmica $\left(l_{s} \gg \mu_{s}\right)$, o sea, cuando $f$ es muchom a yorque $f_{c}\left(f \gg>f_{c}\right)[1]$. L u e go

If $f_{c}>>1$ y $\cosh \left(f f f_{c}\right)>>\cos \left(f f / f_{c}\right)$. Usando la definición del coseno hiperbólico dada por (38), de (34) se puede obtener la expresión para la amplitud de la señal FA en función de $f$ para este régimen, la cual esta dada por (39)

$$
\begin{aligned}
& \cosh \left(\sqrt{f f_{c}^{\prime}}\right)=\frac{e^{\sqrt{f f}}+e^{\sqrt{f f}}}{2} \approx \frac{e^{\sqrt{f^{\prime} f_{c}}}}{2}
\end{aligned}
$$

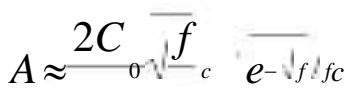

$$
\begin{aligned}
& \text { f }
\end{aligned}
$$

De esta manera en el RTG la amplitud de la señal FA decrece exponencialmente como $/ f$. Además, como la frecuencia de corte $f_{c}$ esta relacionada con la difusividad térmica de la muestra a por (36), podemos determinar el coeficiente $c=f c$ del argumento de la exponencial mediante el ajuste de (39) a los datos experimentales y de este obtenera..

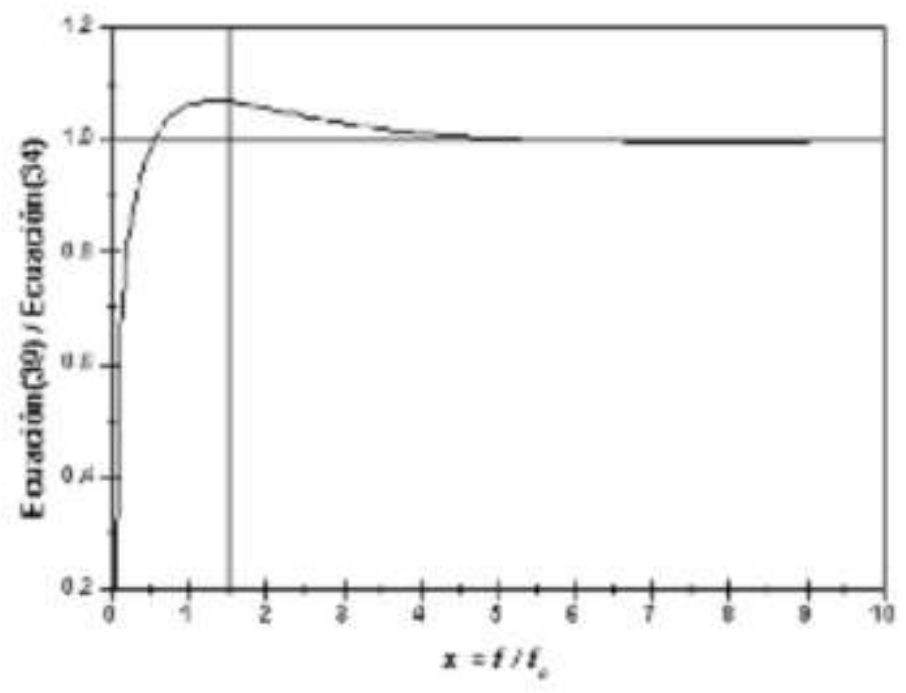

Figura 4. Comparación entre la ecuación (34) y la aproximación (39) para el RTG.

En la figura 4, se muestra la comparación entre las ecuaciones (39) y (34) para la amplitud de la señal FA en función de $x=f / f_{c}$. Se observa, como, para valores de $x>5\left(f>5 f_{c}\right)$ el comportamiento entre las dos expresiones es prácticamente el mismo, en tanto que, para $x>1.5\left(f>1.5 f_{c}\right.$, ) hay un error inferior al $5 \%$ en la aproximación (39).

\section{Conclusiones.}

Se presenta a partir del análisis del modelo RG, el cual utiliza el mecanismo de difusión térmico como principal responsable de la generación de señal FA(ver fig. 3), el análisis de la amplitud de la señal FA en función de la frecuencia de modulación de la radiación incidente,

para la configuración de transmisión de calor (ver fig. 2) en la CFA.

Nuestro resultado dado por la ecuación (34), permite ajustar los datos experimentales de la amplitud de la señal $F A$ en función de $f y$ determinar el parámetro denominado frecuencia de corte o característica de la muestra, luego usando la expresión (36), y conociendo el espesor de la muestra se determina la difusividad térmica de ésta. También presentamos un análisis para el RTF y RTG, del cual podemos inferir que en el primero de éstos 
no es posible utilizar la aproximación dada por (37), para hallar $f_{c}$ al ajustar los datos experimentales, mientras que para el segundo caso, si es posible determinar $f_{c}$ usando la aproximación (39), además, que para valores de $f$ mayores que cinco veces $f_{c}$, el comportamiento de la aproximación dada por (39), es exactamente igual que el presentado para la expresión original dada por (34), en tanto, que para valores de $f$ mayores que uno punto cinco (1.5) veces $f_{c}$, existe un error inferior al $5 \%$ en la aproximación.

En la segunda parte de este trabajo, se presentará la aplicación de esta primera parte, en la determinación experimental de la difusividad térmica de muestras de plata, aluminio, oro, paladio, zinc y cobre entre otras, y así mostrar la potencia de la técnica FA, para determinar experimentalmente este parámetro termofísico de materiales sólidos .

\section{Agradecimientos}

G. Peña Rodríguez, agradece a la Universidad Francisco de Paula Santander en Cúcuta Colombia por su apoyo durante la comisión de estudios de posgrado, y al ICETEX por el crédito otorgado para realizar estudios de posgrado en el extranjero.
1. A. Rosencwaig and A. Gersho, J. Appl.Pys. 47, 64 (1976)

2. A. Rosencwaig, Opt. Commun. 7, 305 (1973)

3. M. J. Adams and G. F. Kirkbright, Analyst, 102, 281-292 (1977).

4. N.F. Leite and L.C. M. Miranda, J. Mat. Sci. 27, 5449 (1992).

5. A. Cruz Orea, I. Delgadillo, H. Vargas, A Gudiño, E. Marín, C. Vásquez-Lopez, A. Calderón and J. J. Alvarado Gil, J. Appl. Phys. 79, 8951 (1996)

6. A. Calderón, J.J. Alvarado Gil, YG. Gurevich, A. Cruz Orea, I. Delgadillo, H. Vargas and L.C.M. Miranda, Physical Review Letters 79, 5022-5025 (1997).

7. C. Vázquez López, A. Calderón, M. Enrique Rodríguez, E. Velasco, S. Canoc, R. Colás and S. Valtierra, J. Mat. Res. 15, 85-91 (2000).

8. YS. Touloukian, RW. Powell, RW HO CY and MC. Nicolu, Thermophysical Propieties of Matter, Vol. 10, Plenum, New York, (1970).

9. G. Ziegler And DPH. Hasselman, Journal of Materials Science. 16, 495-503, (1981).

10. H. Vargas and L.C.M. Miranda, Phys. Rep. 161, 43-101 (1988). 
